\title{
A prevalência da depressão pós-parto e suas consequências em mulheres no Brasil
}

\author{
The prevalence of postpartum depression and its consequences in women in Brazil \\ La prevalencia de la depresión posparto y sus consecuencias en mujeres en Brasil
}

Recebido: 13/11/2021 | Revisado: 21/11/2021 | Aceito: 29/11/2021 | Publicado: 01/12/2021

\author{
Naiana Kimura Palheta de Souza \\ ORCID: https://orcid.org/0000-0001-9292-9041 \\ Universidade Nilton Lins, Brasil \\ E-mail: kimuranay@gmail.com \\ Edivane Queiroz Magalhães \\ ORCID: https://orcid.org/0000-0002-9866-0845 \\ Universidade Nilton Lins, Brasil \\ E-mail: edivanegm_@hotmail.com \\ Omero Martins Rodrigues Junior \\ ORCID: https://orcid.org/0000-0002-8552-3278 \\ Universidade Nilton Lins, Brasil \\ E-mail: omeromartins.farm@gmail.com
}

\begin{abstract}
Resumo
Objetivo: Avaliar a prevalência e os fatores associados para o surgimento da depressão em gestantes no período pósparto. Métodos: foi desempenhada uma verificação crítica das publicações na Scielo, Lilacs e Google Acadêmico, referente aos anos de 2010 a 2021, utilizando-se termos e descritores relacionados à depressão pós-parto. Foram excluídos os artigos que não atenderam aos critérios de inclusão, aqueles que apresentaram duplicidade de dados e os artigos que após leitura detalhada não atenderam ao objetivo proposto nessa revisão. Resultados: foram aparentados, nestes estudos que utilizaram mulheres nos períodos de gravidez, pós-parto imediato e tardio, com ou sem histórico reprodutivo que a DPP é uma condição que deve ser tratada quanto antes para que haja um bom relacionamento familiar, saúde física e mental da criança. Conclusão: A DPP é um transtorno de alta prevalência que pode comprometer a qualidade de relação mãe e a criança, são inúmeros os fatores que contribuem para esse acontecimento como, irritabilidade, sentimento de desamparo e desesperança, a sensação de ser incapaz de lidar com as novas situações, depende de uma série de fatores, o que não permite a realização de um prognóstico baseados em fatores isolados.
\end{abstract}

Palavras-chave: Consequências da depressão; Depressão no pós-parto; Depressão em mulheres; Prevalência da depressão.

\begin{abstract}
Objective: To assess the prevalence and associated factors for the onset of depression in pregnant women in the postpartum period. Methods: a critical review of publications in Scielo, Lilacs and Google Scholar, referring to the years 2010 to 2021, was performed, using terms and descriptors related to postpartum depression. Articles that did not meet the inclusion criteria, those that presented duplicate data and articles that, after detailed reading, did not meet the objective proposed in this review were excluded. Results: in these studies that used women during pregnancy, immediate and late postpartum periods, with or without reproductive history, it was related that PPD is a condition that must be treated as soon as possible so that there is a good family relationship, physical health and of the child. Conclusion: PPD is a highly prevalent disorder that can compromise the quality of the relationship between mother and child. There are numerous factors that contribute to this event, such as irritability, feeling of helplessness and hopelessness, the feeling of being unable to deal with new situations, depends on a series of factors, which does not allow the realization of a prognosis based on isolated factors.
\end{abstract}

Keywords: Consequences of depression; Postpartum depression; Depression in women; Prevalence of depression.

\section{Resumen}

Objetivo: Evaluar la prevalencia y factores asociados para la aparición de depresión en gestantes en el puerperio. Métodos: se realizó una revisión crítica de publicaciones en Scielo, Lilacs y Google Scholar, referidas a los años 2010 a 2021, utilizando términos y descriptores relacionados con la depresión posparto. Se excluyeron los artículos que no cumplieron con los criterios de inclusión, los que presentaron datos duplicados y los artículos que, luego de una lectura detallada, no cumplieron con el objetivo propuesto en esta revisión. Resultados: en estos estudios que utilizaron mujeres durante el embarazo, posparto inmediato y tardío, con o sin antecedentes reproductivos, se relacionó que la DPP es una condición que debe ser tratada lo antes posible para que exista una buena relación familiar, salud física y del niño. Conclusión: La DPP es un trastorno de alta prevalencia que puede comprometer la 
calidad de la relación madre-hijo. Son numerosos los factores que contribuyen a este evento, como la irritabilidad, la sensación de desamparo y desesperanza, la sensación de no poder afrontar nuevos problemas. situaciones, depende de una serie de factores, lo que no permite la realización de un pronóstico basado en factores aislados.

Palabras clave: Consecuencias de la depresión; Depresión post-parto; Depresión en mujeres; Prevalencia de la depresión.

\section{Introdução}

O estresse é um conjunto de reações para que o corpo emita uma reação ao que despertou. Quando o organismo fica vulnerável diante as situações de perigo ou ameaça, o corpo começa a entrar em estado de alerta, provocando alterações físicas e emocionais, fazendo assim com que o corpo passe a funcionar com maior consumo de energia. Como consequência de stress crônico, o surgimento de outros distúrbios como, ansiedade e depressão apresentam-se no indivíduo. Alterações importantes no cotidiano da vida podem resultar respostas de stress (Rodrigues \& Schiavo, 2011).

Embora a gravidez seja considerada um período fisiológico normal para as mulheres, é assimilado com grande importância, que podem ter alterações comportamentais e biológicas, podendo interferir nas relações entre mãe, família e amigos, interferindo também no elo entre mão e bebê podendo desenvolver a depressão (Alves \& Silva, 2021).

Atividades estimulantes de sinais afetivos como conversar, ler e cantar entre mãe e bebê tem sua importância e eficácia na construção de vinculo mesmo antes de nascer. Essa prática, através de toques, cuidados com corpo, o diálogo maternal, induz o bebê emanar gradativamente as suas primeiras expressões comunicativas no diálogo (Carlesso \& Souza, 2011).

O pós-parto é um espaço de tempo delicado na vida da mãe por envolver alterações físicas e psíquicas podendo influir diretamente na saúde psicológica, ascendendo o risco de desenvolvimento de distúrbios psiquiátricos. Estudos direcionam a repercussão a curto e longo prazo que o período puerperal exerce na vida da mãe, afetando principalmente na saúde mental e o impacto negativo ao seu redor. Visto que o relacionamento da díade fica dificultada, dificultando no período de amamentação, o que é de grande importância na saúde e desenvolvimento da criança, onde filhos de mães depressivas são passíveis a doenças relacionadas a distúrbios nutricionais, problemas no desenvolvimento emocional, cognitivo e social (Abuchaim et al.,2016).

Sendo assim, o objetivo deste estudo foi identificar, através de uma revisão bibliográfica, os fatores de risco e sintomas considerados determinantes para o surgimento de depressão em gestantes no período pós-parto e suas consequências na vida da criança.

\section{Metodologia}

A metodologia desenvolvida trata-se de revisão bibliográfica descritiva, de modo qualitativa acerca do tema. Foram realizadas buscas de pesquisas cientificas nas bases de dados Scielo (Biblioteca Eletrônica Scientific Eletronic Library Online), Lilacs (Literatura Latino-americana e do Caribe em Ciências da Saúde) e Google acadêmico, usando como critério de seleção artigos publicados entre 2011 a 2021, publicações em português, artigos condizentes com o tema abordado são com as seguintes palavras-chaves: depressão pós-parto, período pós-parto, transtornos pós-parto. Critérios de exclusão, artigos que estão fora do tema e tempo proposto, artigos repetidos, artigos com conteúdo divergentes. Com objetivo de realizar uma revisão bibliográfica em relação aos sintomas depressão pós-parto e suas consequências na vida da criança.

Os artigos foram analisados em três etapas:

- Primeira etapa: verificação de artigos nas bases de dados seguindo o contexto de título, resumo, palavras chaves e exclusão dos que não se encaixam nos critérios estabelecidos.

- Segunda etapa: seleção dos artigos que se encaixam nos critérios de inclusão para uso no desenvolvimento pesquisa. 
- Terceira etapa: leitura dos artigos e seleção dos que apresentam o assunto adequado para esta pesquisa.

Figura 1. Fluxograma de critérios de exclusão e inclusão.

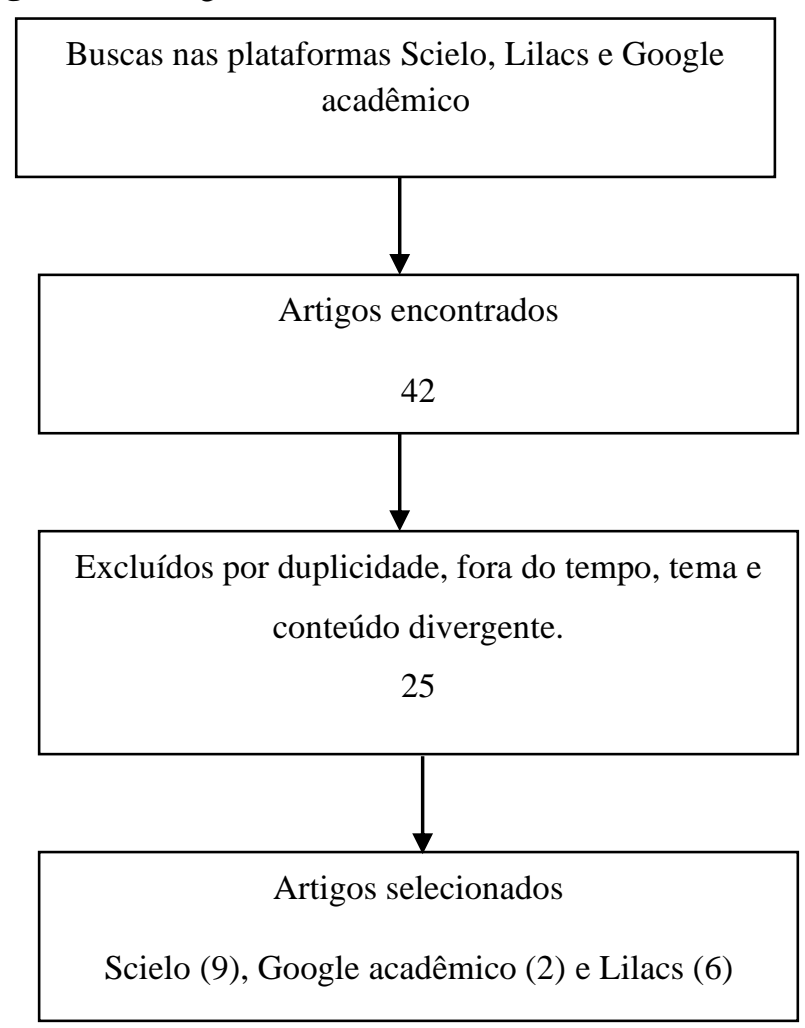

Fonte: Autores (2021).

\section{Resultados e Discussão}

O Quadro 1, demonstra as características dos principais artigos selecionados, enfatizando os fatores e sintomas da depressão pós-parto. 
Quadro 1. Características dos principais artigos selecionados.

\begin{tabular}{|c|c|c|c|}
\hline Autor, Ano & Título & Objetivos & Principais resultados \\
\hline $\begin{array}{l}\text { Alves \& } \\
\text { Silva, 2021. }\end{array}$ & $\begin{array}{l}\text { Depressão pós-parto } \\
\text { e seus efeitos na } \\
\text { relação mãe-bebê. }\end{array}$ & $\begin{array}{l}\text { Analisar as características da depressão } \\
\text { pós-parto e fatores de risco associados à } \\
\text { sua ocorrência. Bem como: } \\
\text { Interferência na relação mãe-bebê. }\end{array}$ & $\begin{array}{l}\text { Compreende-se que não existe uma única causa } \\
\text { conhecida para depressão pós-parto. Ela pode estar } \\
\text { associada a fatores físicos, emocionais, estilo e } \\
\text { qualidade de vida, além de ter ligação, também, com } \\
\text { histórico de outros problemas e transtornos mentais }\end{array}$ \\
\hline $\begin{array}{l}\text { Arrais, } \\
\text { Araújo } \quad \& \\
\text { Schiavo, } \\
2018 .\end{array}$ & $\begin{array}{l}\text { Fatores de risco e } \\
\text { proteção associados } \\
\text { à depressão pós- } \\
\text { parto no pré-natal } \\
\text { psicológico }\end{array}$ & $\begin{array}{l}\text { Identificar fatores de risco e de proteção } \\
\text { associados à Depressão Pós-Parto } \\
\text { (DPP); e avaliar a contribuição do Pré- } \\
\text { Natal Psicológico (PNP) como } \\
\text { programa de prevenção em Saúde da } \\
\text { Mulher }\end{array}$ & $\begin{array}{l}\text { Verificou-se tal associação quanto a gravidez não } \\
\text { planejada e a falta de apoio do pai do bebê, } 10,64 \% \text { das } \\
\text { puérperas revelaram tendência para DPP. considera-se } \\
\text { que o PNP, somado a fatores de proteção, constitui ação } \\
\text { preventiva a ser desenvolvida pelo profissional de } \\
\text { Psicologia no contexto do acompanhamento pré e pós- } \\
\text { natal. }\end{array}$ \\
\hline $\begin{array}{l}\text { Moll et al., } \\
2019\end{array}$ & $\begin{array}{l}\text { Tracking } \\
\text { Postpartum } \\
\text { depression } \\
\text { Young wonen }\end{array}$ & $\begin{array}{l}\text { Rastrear a depressão pós-parto entre } \\
\text { mulheres jovens que estão na segunda } \\
\text { semana e no sexto mês após o parto. }\end{array}$ & $\begin{array}{l}\text { Evidencia-se que a depressão pós-parto precisa ser } \\
\text { investigada na atenção primária em saúde, que deve } \\
\text { valorizar os aspectos sociodemográficos e individuais } \\
\text { para estabelecer um plano de cuidados integral desde o } \\
\text { pré-natal, com vistas à prevenção desse frequente } \\
\text { transtorno do puerpério. }\end{array}$ \\
\hline $\begin{array}{l}\text { Rodrigues et } \\
\text { al., } 2019\end{array}$ & $\begin{array}{l}\text { Consequências da } \\
\text { depressão pós-parto } \\
\text { no desenvolvimento } \\
\text { infantil: revisão } \\
\text { integrativa. }\end{array}$ & $\begin{array}{lccr}\text { Identificar as consequências } & \text { da } \\
\text { depressão pós-parto para } & \text { o } \\
\text { desenvolvimento infantil. } & & \end{array}$ & $\begin{array}{l}\text { A depressão pós-parto merece maior atenção a nível de } \\
\text { saúde pública. Assim estratégias precoces de } \\
\text { intervenção para mães como características de depressão } \\
\text { pós-parto são necessárias para assegurar o bem-estar } \\
\text { mental das mulheres e contribuir para um } \\
\text { desenvolvimento pueril saudável. }\end{array}$ \\
\hline $\begin{array}{l}\text { Rodrigues \& } \\
\text { Schiavo, } \\
2011 .\end{array}$ & $\begin{array}{l}\text { Stress na gestação e } \\
\text { no puerpério: uma } \\
\text { correlação com a } \\
\text { depressão pós-parto. }\end{array}$ & $\begin{array}{l}\text { Descrever e comparar as fases do stress } \\
\text { de primigestas no terceiro trimestre de } \\
\text { gestação e no pós-parto e correlacioná- } \\
\text { las à ocorrência de depressão pós-parto } \\
\text { (DPP). }\end{array}$ & $\begin{array}{l}\text { Tanto na gestação como no puerpério mais da metade } \\
\text { das mulheres apresentam sinais significativos para } \\
\text { stress. Entretanto, a frequência da manifestação dos } \\
\text { sintomas significativos de stress na gestação foi superior } \\
\text { à frequência apresentada no puerpério. Tais resultados } \\
\text { parecem guardar uma estreita relação com a } \\
\text { manifestação de DPP, indicando relação entre stress e } \\
\text { DPP. }\end{array}$ \\
\hline $\begin{array}{l}\text { Ramos et al., } \\
2018 .\end{array}$ & $\begin{array}{l}\text { Fatores associados à } \\
\text { depressão pós-parto: } \\
\text { revisão integrativa }\end{array}$ & $\begin{array}{l}\text { Identificar os fatores determinantes para } \\
\text { o surgimento de depressão em gestantes } \\
\text { no período pós-parto. }\end{array}$ & $\begin{array}{l}\text { Os sofrimentos emocionais em conjunto ao maior } \\
\text { número de estressores vivenciado e a depressão pré- } \\
\text { natal pode ser apontado como fatores para o surgimento } \\
\text { da depressão pós-parto. }\end{array}$ \\
\hline
\end{tabular}

Fonte: Autores.

Diante dos resultados encontrados, foram categorizados abaixo os fatores de risco, diagnóstico, as consequências para a mãe e para a criança, o tratamento e a profilaxia sobre a DPP.

\subsection{Fatores que exercem influência no desenvolvimento de depressão pós-parto}

A depressão pós-parto (DPP) é um transtorno psiquiátrico que evolui negativamente sobre a paciente que se estende a criança e as relações familiares, podendo prevalecer por anos e está ligada ao aumento de perigos de outros quadros depressivos recorrentes. Devido à limitação que a doença impede a mãe de responder à demanda envolvida nos cuidados e sua relação maternal a criança, o impacto sobre ela é significativo, filhos de mulheres deprimidas são susceptíveis a doenças diarreicas, distúrbios nutricionais e alterações no desenvolvimento físico, emocional, cognitivo e social. Na visão etiológica existem simultaneamente dois modelos explicativos possivelmente complementares, um biológico que suste a existência de uma vulnerabilidade hormonal ou genética, e o outro modelo psicossocial, que move as transformações na vida da mulher pósparto, favoreçam o desenvolvimento da depressão (Figueira, Diniz \& Silva, 2011) (Abuchaim et al., 2016).

Entre os vários fatores de risco que estão relacionados com as alterações no período de DPP, estão: gravides na adolescência ou idade inferior a 16 anos, histórico de transtorno psiquiátrico prévio, episódios de estresse nos últimos 12 meses, conflitos conjugais, estado civil podendo ser solteira ou divorciada, desemprego que tanto pode ser a puérpera como seu cônjuge e a ausência oi insuficiência de suporte social. Envolve ainda a espera de um bebê do sexo oposto ao desejado e um 
suporte emocional deficiente com relações insatisfatória (Ramos et al.,2018). A falta de apoio durante a gestação, seja da família, do companheiro ou de amigos, aumenta o risco para a ocorrência de depressão (Hartmann, Mendoza-Sassi \& Cezar, 2017).

Para Alves et al. (2017), outros tópicos podem ser considerados fatores de risco para DPP em mulheres que engravidam após os 34 anos de idade, no qual são as complicações obstétricas em decorrência do envelhecimento ovariano e também a pré-existência de doenças crônicas que aparecem ao longo da idade, observando que essas mulheres apresentam maior frequência de complicações perinatais adversas, destacando-se a prematuridade, baixo peso ao nascer, hipertensão/préeclâmpsia.

Ramos et al. (2018), declaram que durante o pós-parto, a conjuntura psiquiátrica mais comum é a depressão pós-parto que na escala mundial é um problema de saúde pública e embora uma quantidade grande de mães que são usuárias da atenção primária de saúde, ainda sim os transtornos depressivos geralmente não são detectados ou tratados, apesar da disponibilidade de tratamentos eficazes. O quadro clínico manifesta-se em grande parte dos casos, a partir das primeiras quatro semanas pósparto e consegue atingir a sua potência máxima nos seis primeiros meses. Sendo a depressão a principal causa de problemas de saúde e incapacidade em todo o mundo, é uma doença de diagnóstico complexo que tem dado preocupação aos profissionais da saúde pois é uma principal causa de incapacitação em todo mundo e até 2030, estima-se que tornará a doença mais prevalente no planeta.

Para diagnosticar a DPP, pode incluir o quadro clinico da paciente, associando pelo menos cinco sintomas, com a duração mínima de duas semanas, segundo do Manual de Diagnóstico e Estatística das Perturbações Mentais (DSM-IV), que compõem: humor deprimido, ausência de capacidade, mudanças consideráveis no peso e do apetite, insônia, agitação ou retardo psicomotor, fadiga, sensação de inutilidade ou culpa, capacidade diminuída de pensar, de concentrar-se, insegurança e pensamentos suicidas (Oliveira \& Dunningham, 2015).

A depressão pode ter abrangência entre mulheres com as mais diferentes classes sociais, cor e raça, todavia, as mais predispostas são aquelas com maior vulnerabilidade social de pobreza e com a escassez de apoio psicológico, ficando mais suscetíveis as primíparas de baixa renda (Moll et al., 2019).

Para Brito et al. (2015), a DPP é considerada um transtorno comum com fator de risco para o suicídio muito forte, que é caracterizado por tristeza insistente, a falta de capacidade ou ânimo para realização de atividades cotidianas. Com meios de informações, ofertas de métodos contraceptivos a gravidez indesejada pode ser evitada podendo reduzir a chance de uma depressão pós-parto. É importante na rotina de atenção primária e saúde materna a existência de programas de políticas públicas, que visem estratégias de enfrentamento com equipe multidisciplinar para a identificação precoce dos sintomas para assim fazer um tratamento especifico imediato.

Conforme Arrais, Araújo e Schiavo (2018), a complicação de maior existência no período pós-parto é a depressão, podendo atingir $15 \%$ a $20 \%$ das puérperas. A definição de fatores de risco e proteção podem colaborar para melhor entendimento da doença e para a formação de estratégias de prevenção e diagnóstico precoce da patologia (Figueira, Diniz \& Silva, 2011).

\subsection{Consequências para a mãe e a criança}

Para Rodrigues et al. (2019), como consequência dos impactos da DPP, a baixa responsiva da mãe com o diagnóstico pode ter efeitos insatisfatórios no desenvolvimento infantil, como resultar em desordens linguísticas, físicas, cognitivas e sociais, além do abandono da amamentação, influenciando na alimentação da criança e em escores nutricionais inferiores. Vale destacar que são muitas as complicações na relação mãe-bebê na DPP, podendo dividir as mais importantes em três categorias: ambivalência afetiva na díade, dificuldades maternas na amamentação e instabilidade no sono. A intimidade da 
díade nos primeiros anos de vida da criança reflete no desenvolvimento infantil, o comportamento de estimulação de relacionamento é essencial para crescimento adequado físico e emocional da criança, a presença de uma mãe com comportamentos depressivos e pouco responsiva pode gerar déficits na interação da díade.

A regulação emocional, autocontrole e a interação da criança com o mundo estão sujeitos à forma de como essa relação mãe-bebê é estabelecida (Campos \& Rodrigues, 2015).

Existe uma relação direta entre a DPP e amamentação, o abandono do aleitamento materno, já que a amamentação é gesto de amor, carinho e admiração, inicio de ciclo da mulher como mãe e quando não conseguem atender as necessidades fisiológicas do bebê, provoca um sentimento de frustação e nervosismo em relação ao exercício da maternidade. Esse sentimento de incapacidade materna é responsável também pela dificuldade na produção do leite e como consequência, na amamentação, implicando assim na vida nutricional do bebê. Outro importante fator a ser cuidado é a de instabilidade no sono dos bebês, caracterizado como leve, agitado e de curta duração, aspectos influenciados pela doença das mães, pois é real que as dificuldades desse padrão estão ligadas às condições emocionais de seu cuidador. Isso simboliza que o bebê tem dificuldade em encontrar sua mãe, como alguém que lhe proporcione segurança uma vez que a mesma não tem autoconfiança nos cuidados maternos (Rodrigues et al., 2019).

Para o rastreamento dos sintomas que se apresentam no pós-parto, usa-se a escala de auto avaliação de Edimburgh (EDPS), que compreende em um questionário de auto avaliação com esquemas de perguntas com quatro opões, que de acordo com a presença ou intensidade dos sintomas são pontuadas de 0 a 3. Quando o resultado da pontuação de repostas for igual ou maiores que 10, as entrevistadas são consideradas como do grupo de risco para desenvolver a depressão e é indicado procurar o especialista para fazer o acompanhamento profissional cabível (Lima et al, 2016).

De Oliveira e Dunningham (2015), a simplicidade e agilidade da EPDS, no qual não exige muito tempo para seu preenchimento, devem ser inseridos em níveis de atenção primária evitando sub-diagnósticos, promove-se essencial para uso de rotina clínica por profissionais que não são especializados em saúde mental fazer o rastreamento de mães que apresentam sintomas de depressão e assim não sobrecarregando o serviço especializado e detectando precocemente a patologia, devem ser inseridos.

O profissional de saúde é a pessoa mais indicada em estimular a mulher venha a desenvolver habilidades para desempenhar o autocuidado, melhoria na sua condição de saúde, realizando o monitoramento do período da gestação através de atendimento humanizado, visto que esse tipo de atendimento, o aumento do vínculo materno será estimulado contribuindo assim, para uma prevenção e em um diagnóstico precoce de depressão pós-parto. A equipe de saúde deverá estabelecer com a paciente um elo confiável observando seus comportamentos, em casos de pacientes que estejam deprimidas, estimular com atividades construtivas e cuidados pessoais (Arrais, Mourão \& Fragalle, 2014).

Como forma de profilaxia na DPP, é o pré-natal psicológico, que vem inovando com o conceito em atendimento perinatal aprimorado, humanizado no processo gestacional. O programa tem como o objetivo a integração da gestante e da família a todo o processo da gravidez e pós gravidez, com encontros temáticos em grupos, enfatizando a psicoterapêutica na preparação psicológica dos pais e a prevenção da DPP. O PNP (pré-natal psicológico), vem complementar o tradicional prénatal, trazendo um perfil psicoterapêutico, oferecendo apoio emocional, colocando em pauta soluções para demandas que poderão surgir no período gravídico-puerperal, como por exemplo: mitos relacionados a maternidade, gestação de risco, à malformação do feto, medo do parto, transtornos depressivos e de ansiedade, às mudanças de papéis familiares e sociais, às alterações na libido, entre outros (Arrais, Mourão \& Fragalle, 2014).

O programa incentiva ainda ás gestantes a terem um relacionamento com outras mulheres através de grupos que tenham em comum sentimentos e emoções presentes nessa fase, ajudando assim a identificar os sentimentos presentes. $\mathrm{O}$ encontro tem como objetivo acolher e dar voz as mesmas, orientar e instruir para que passem por esse processo de maneira 
mais suave possível (Arrais, Mourão \& Fragalle, 2014).

\section{Considerações Finais}

A DPP, se não tratada apropriadamente, tem uma chance maior de se desenvolver, durar meses e até tornar-se em um distúrbio depressivo crônico. Mesmo com todos os cuidados necessários, a depressão pós-parto aumenta o risco de futuras ocorrências depressivas, o que demanda um acompanhamento periódico da saúde mental da pessoa. Se não for tratada da forma correta e de forma imediata, a DPP pode interferir negativamente o vínculo entre mãe-filho e acarretar problemas familiares, muitos deles irreversíveis. Filhos de mães que têm DPP não tratada são mais sujeitos a ter problemas de comportamento, como privação para dormir e comer, crises de birra e hiperatividade, levando em consideração a falta de diálogo que é primordial para uma boa convivência familiar. Os atrasos no desenvolvimento da linguagem são mais comuns também.

A mulher que está em DPP, normalmente, amamenta pouco, deixando assim a criança com falta de nutrientes essências do leite materno e não cumpre o calendário de vacinas dos bebês e assim prejudicando a saúde da criança. Por sua vez, as crianças apresentam maior risco de desenvolver baixo peso e distúrbios psicomotores, e os custos emocionais associados à DPP reduzem as interações mãe-filho. Também podem desenvolver sintomas como irritabilidade, choro frequente, desesperança, sensação de desproteção, redução da energia e motivação, falta de interesse por sexo, distúrbios alimentares e do sono, ansiedade e incapacidade de lidar com novas situações são emocionalmente redobradas. É importante atentar que, em casos mais graves a depressão pós-parto pode levar ao suicídio. De forma geral, o que pode ser feito para prevenir a depressão pós-parto, é cuidar da saúde mental.

Finalizando, este presente trabalho fica em sugestão para pesquisas futuras, nesse contexto para o fortalecimento do tema enfatizando na importância do acompanhamento profissional em mães com sintomas de depressão pós-parto. Fatores como convivência familiar e social contribuem para o desenvolvimento dessa patologia.

\section{Referências}

Abuchaim, E. S., Caldeira, N. T., Di Lucca, M. M., Varela, M. \& Silva, I. A. (2016). Depressão pós-parto e autoeficácia materna para amamentar: prevalência e associação. Acta Paulista de Enfermagem, 29(6), 664-670. https://doi.org/10.1590/1982-0194201600093

Alves, B. K. G. \& Silva, E. G. (2021). Depressão pós-parto e seus efeitos na relação mãe-bebê. Revista de Iniciação Cieníficat e Extensão, 4(1), 536-547.

Alves, N. C. de C., Feitosa, K. M. A., Mendes, M. E. S. \& Caminha, M. de F. C. (2017). Complications in pregnancy in women aged 35 or older. Revista Gaácha de Enfermagem, 38(04), 1-8.

Arrais, A. da R., Araujo, T. C. C. F. \& Schiavo, R. A. (2018). Fatores de risco e proteção associados à depressão pós-parto no pré-natal psicológico. Psicologia: Ciência e Profissão, 38(4), 711-729.

Arrais, A. R., Mourão, M. A. \& Fragalle, B. (2014). O pré-natal psicológico como programa de prevenção à depressão pós-parto. Sáude e Sociedade, 23(1), 251-564.

Brito, C. N. O., Alves, S. V., Ludermir, A. B. \& Araújo, T. V. B. (2015). Depressão pós-parto entre mulheres com gravidez não pretendida. Revista Saúde Pública, 49(33), 1-9.

Campos, B. C. \& Rodrigues, O. M. P. R. (2015). Depressão Pós-Parto Materna: Crenças, Práticas de Cuidado e Estimulação de Bebês no Primeiro Ano de Vida. Psico, Porto Alegre, 46(4), 483-492.

Carlesso, J. P. P. \& Souza, A. P. R. (2011). Dialogia mãe-filho em contextos de depressão materna: revisão de literatura. Revista CEFAC, 13(6), 119-1126. https://doi.org/10.1590/S1516-18462011005000085

Hartmann, J. M., Mendoza-Sassi, R. A. \& Cesar, J. A. (2017). Depressão entre puérperas: prevalência e fatores associados. Cadernos de Saúde Pública, 33(9), $1-10$.

Figueira, P. G., Diniz, L. M. \& Silva, H. C., Filho. (2011). Características demográficas e psicossociais associadas à depressão pós-parto em uma amostra de Belo Horizonte. Revista de Psiquiatria do Rio Grande do Sul, 33(2), 71-75.

Lima, N. C., Ravelli, A. P. X., Messias, L. S. F. \& Skupien, A. V. (2016). DEPRESSÃO PÓS-PARTO BASEADA NA ESCALA DE EDIMBURGO. Revista Conexão UEPG, 12(2), 268-277. 
Research, Society and Development, v. 10, n. 15, e597101523272, 2021

(CC BY 4.0) | ISSN 2525-3409 | DOI: http://dx.doi.org/10.33448/rsd-v10i15.23272

Moll, M. F., Matos, A., Rodrigues, T. A., Martins, T. S., Pires, F. C. \& Pires, N. A. S. (2019). Tracking Postpartum depression in Young wonen. Journal of Nursing, UFPE online, 13(5), 1338-1344. https://doi.org/10.5205/1981-8963-v13i05a239289p1338-1344-2019

Oliveira, M. J. M. \& Dunningham, W. A. (2015). Prevalência e fatores de risco relacionados a depressão pós-parto em Salvador. Revista Brasileira de Neurologia e Psiquiatria, 19(2), 72-83.

Ramos, A. S. M. B, Martins, A. C. Q., Pessoa, D. L. R., Machado, M. C. A. M. \& Noronha, F. M. F. (2018). Fatores associados à depressão pós-parto: revisão integrativa. Enciclopédia Biosfera, 15(27), 4-13.

Rodrigues, O. M. P. R. \& Schiavo, R. A. (2011). Stress na gestação e no puerpério: uma correlação com a depressão pós-parto. Revista. Brasileira de Ginecologia e Obstetrícia, 33(9), 252-257. https://doi.org/10.1590/S0100-72032011000900006

Rodrigues, W. L. C., Branco, J. G. O., Facundo, S. H. B. C., Costa, F. B. C. \& Oliveira, C. J. (2019). Consequências da depressão pós-parto no desenvolvimento infantil: revisão integrativa. Revista Nursing, 22 (250): 2728-2733 